\title{
The Method of Structural Damage Identification by ACO Algorithm and Wavelet Theory
}

\author{
Deqing Guan *, Jun Tang and Tao He \\ School of Civil Engineering and Architecture,Changsha University of Science and Technology, Changsha, China \\ ${ }^{*}$ Corresponding author
}

\begin{abstract}
In order to effectively identify the location and extent of structural damage, a two-stage damage identification method, ACO algorithm and wavelet theory were presented. First, the curvature mode of structure with damage was obtained with finite element analysis. The curvature modes were analyzed using continuous wavelet transform by Mexh wavelet on multiple scales, and the location of the damage could be identified by the maximum of the wavelet coefficients. Then, the objective function was established by the frequency and modal shape. ACO algorithm was used to solve constrained optimization problems, to identify the degree of structure damage. Through the analysis to a three-span continuous beam, the results show that the ACO algorithm and wavelet theory can effectively identify the damage location and damage degree of the structure.
\end{abstract}

Keywords-wavelet; ACO algorithm; damage identification; finite element method

\section{INTRODUCTION}

Continuous beam members are commonly used in bridge structures, structural damage exists due to natural and human factors inevitably. Structural safety was threatened, so it is important to study the damage identification method of continuous beam structure.

French earth physicist J. Morlet proposed the concept of wavelet transform in 1974 . Wavelet transform is a time and frequency local transformation, and it can withdraw the information from the signal. As a new signal processing method, wavelet analysis has the characteristic spatial localization. Wavelet transform signal at a point is completely determined by the local information near the point on the low scale, so it can analyze the singularity position of the signal. In recent years, wavelet analysis method has been gradually applied to diagnose structural damage. Liew etc [1] discrete wavelet transform was used to identify the crack location of a simply supported beam. Raghavendrachar etc[2] established a three-span concrete continuous beam model with its curvature mode damage detection and proved curvature mode of local damage is very sensitive and reliable. M. Rucka and K. Wilde etc [3] adopted one-dimensional continuous wavelet to analysis a cantilever beam with damage model, and used twodimensional wavelet to analysis four edges fixed plate model. The results show that the corresponding damage location where the wavelet mathematical model arrive at the maximum point and where it insured by the wavelet analysis method are not dependent on the characteristics of the structure and mathematical model. Guan Deqing [4] put forward that the problem of frame structure damage identification of strain mode can be solved by using wavelet transform. The results show that the method is able to identify damage of frame structure. Damage identification of continuous steel box girder with cracks was carried out using curvature mode continuous wavelet transform by Shi etc [5].

ACO algorithm was mentioned by Dr. Marco Dorigo [6 8]at first, his colleagues studied by long term. ACO is the simulation of ant community food-gathering process, successfully applied to many discrete optimization problems such as: the traveling salesman problem (TSP), quadratic assignment problems, planning problems, network dynamic routing problems. Gutjahr W J[9] proved an improved ant colony algorithm theoretically from the point of view of directed graph theory for the first time. Recently, ACO algorithm also began to be applied to a small number of optimization problems of continuous function. In this paper, damage problem was transformed into constrained optimization by ACO algorithm.

\section{APPLICATION OF ACO AlgORITHM AND WAVELET THEORY IN STRUCTURAL DAMAGE IDENTIFICATION}

A. Identification of Structural Damage Location by Wavelet Transform

Wavelet transform is defined an indigestion of basic wavelets functions $\psi(t)$ after displacement $\tau$ and the signal $x(t)$ at different scales $a$ which is to be analyzed:

$$
W T_{x}(a, \tau)=\frac{1}{\sqrt{a}} \int_{-\infty}^{\infty} x(t) \psi^{*}\left(\frac{t-\tau}{a}\right) d t, a>0
$$

$a$ in equation (1) is a scale factor, reflecting signal frequency information. $\tau$ is shift factor reflecting signal time information. $a, \tau, t \in R$ and $a \neq 0 . \psi *(t)$ shows the complex conjugate of $\psi(t)$. With the conversion of $a, t$, the wavelet transform has multi-resolution features.

From mechanics theory, the curvature of the beam can be expressed as:

$$
\rho=\frac{M}{E I}
$$




$$
\rho=\frac{\partial \theta}{\partial x}=\frac{\partial^{2} y}{\partial x^{2}}
$$

Where in: $M$ is a cross-sectional moment; $E$ is the modulus of elasticity; $I$ is a cross-sectional moment of inertia; $\theta$ and $y$ denote the angle of the beam and displacement modes.

Bring (2) into (3) :

$$
\rho=\frac{M}{E I}=\frac{\partial^{2} y}{\partial x^{2}}=\frac{y_{i+1}-2 y_{i}+y_{i-1}}{l^{2}}=\phi_{n}^{\prime \prime}(x)
$$

$i$ is the $i$-th measuring points; $l$ is the distance between the two measuring points; $\psi_{n}^{\prime \prime}(x)$ is n-th order beam curvature mode shape.

From (4), local damage will led to structural decline in local stiffness, curvature mode shape mutating, location curvature mode discontinuing and existing a singular point. According to singularity detection theory, wavelet transform coefficients of curvature mode appears local modulus maximum on lesions, the position of damage can be determined by the position of wavelet modulus maximum position. The curvature modes were analyzed using continuous wavelet transform on multiple scales, and the location of the crack could be identified by the maximum of the wavelet coefficients.

\section{B. Identification of the Extent of Structural Damage Based on ACO}

Dynamic characteristic equation of a system of $n$ degrees of freedom and its stiffness matrix can be expressed as:

$$
\begin{gathered}
k \varphi_{j}=\lambda_{j} M_{0} \varphi_{j} \\
K=K_{0}+\sum_{i=1}^{N_{e}} T_{i} K_{i}
\end{gathered}
$$

$K_{0}$ and $K$, are the total stiffness of the structure before and after damage, respectively. $M_{0}$ is the total mass of the intact structure. $\lambda_{j}$ and $\varphi_{j}$, respectively, are the n-th order equation and eigenvalue feature vectors. $\lambda_{j}=\left(2 \pi f_{j}\right)^{2}, f_{j}$ is the J-th order natural frequency $(\mathrm{Hz}) ; j=1,2,3 \cdots N_{m}\left(N_{m}\right.$ is the measured modal order); $K_{i}$ is the i-th single element stiffness matrix unit extended order, $i=1,2,3 \cdots N_{e}\left(N_{e}\right.$ is total unit of structure finite element model); $\alpha=\left[T_{1}, T_{2}, T_{3} \cdots T_{N_{e}}\right]^{T}$ as the coefficient of degree of unit damage. The objective function is defined as a form of non-Linear least squares with boundary constraints:

$$
\min _{T \in R}\left\{f_{T}=\frac{1}{2}\|r(T)\|^{2}=\frac{1}{2} \sum_{i=1}^{N} r_{i}^{2}(T) \mid \text { low } \leq T \leq u p\right\}
$$

up and low respectively are the lower and upper bounds of T. To meet identification requirements, define low $=0$, up $=1\left(j=1,2,3 \cdots N_{e}\right)$, when $T=0$ damage is zero, when $T=1$ it is completely damaged. N-dimensional target function vector consists of frequencies and modes, as follow:

$$
\begin{gathered}
r(T)_{N \times 1}=\left[r_{f}(T)_{N_{m}}^{T}, r_{h}(T)_{N_{m}}^{T}\right]^{T} \\
r_{f}^{i}(T)=\left|1-\frac{f_{a}^{i}(T)}{f_{t}^{i}}\right| \\
r_{h}^{i}(T)=1-\frac{\left|\left(\varphi_{t}^{i}\right)^{T} \varphi_{a}^{i}(T)\right|^{2}}{\left|\left(\varphi_{t}^{i}\right)^{T} \varphi_{t}^{i}\right|\left|\varphi_{a}^{i}(T)^{T} \varphi_{a}^{i}(T)\right|}
\end{gathered}
$$

Subscript $\mathrm{t}$ and $\mathrm{a}$ are the measured value and analysis value. $r_{f}^{i}(T)$ and $r_{h}^{i}(T)$ are Close value of the measured value and the value of analysis which is from MAC value of natural frequencies and modes. In this paper, the structure of frequency and vibration data was used to solve constrained optimization problems described above by AOC, to achieve recognition of the extent of damage of structure.

\section{NumERICAL Simulation ANALySis}

A three-span continuous beam structure finite element model which contains damage shown in Figure I. Sectional dimension: $\quad b \times h=200 \mathrm{~mm} \times 250 \mathrm{~mm} \quad ; \quad$ Material density $\quad \rho=7800 \mathrm{~kg} / \mathrm{m}^{3} \quad ; \quad$ Elastic Modulus: $E=2.1 \times 10^{11} \mathrm{~N} / \mathrm{m}^{2}$; Poisson's ratio: $\mu=0.3$. The whole frame structure is divided into 600 units. Beam elements were designated number 1-600 by the sequence of A-B-C-D.

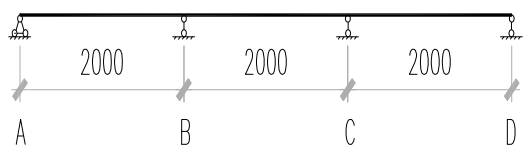

\section{FIGURE I. CONTINUOUS BEAM FINITE ELEMENT MODEL $(\mathrm{mm})$}

Structural damage was simulated by reducing the element stiffness. Condition was assumed in this article, as shown in Table I.

TABLE I. Continuous BeAm Damage CASES

\begin{tabular}{c|l|l}
\hline Damage Cases & Damage Location & Damage Extent \\
\hline \multirow{3}{*}{ Condition 1 } & $30^{\text {m }}$ unit & $20 \%$ \\
\cline { 2 - 3 } & 180 th unit & $20 \%$ \\
\cline { 2 - 3 } & $260^{\text {th }}$ unit & $30 \%$ \\
\hline
\end{tabular}




\section{A. Identification of Structural Damage Location by Wavelet Analysis}

Finite element model of the structure was built, structural damage curvature mode was calculated. Selecting Mexh wavelet as mother wavelet, wavelet coefficients was obtained by doing Continuous wavelet transform to curvature modal of structure (as shown in Figure II). Three singular points (30,180 and 260) were found in wavelet figure. Mutation corresponds exactly to the position of damaged cell of structure.

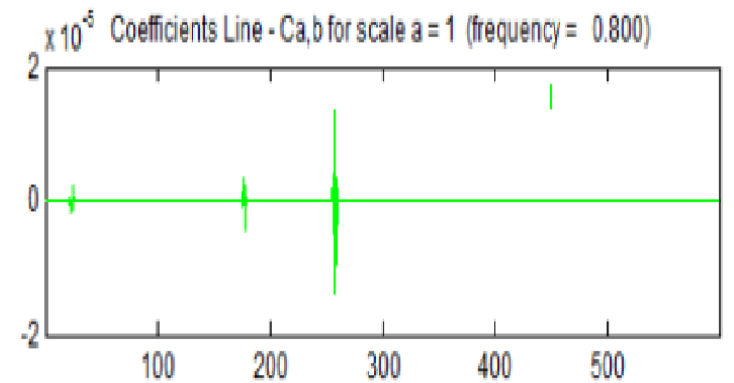

FIGURE II. WAVELET COEFFICIENTS OF CONTINUOUS BEAM WITH THREE DAMAGES

\section{B. Identification of the Extent of Structural Damage By ACO}

The extent of structural damage was identified by 10-order frequency and the first-order vibration modal.(as shown in figure III).The damage degree of unit 30 is $15 \%$,the damage degree of unit 180 is $20 \%$, the damage degree of unit 260 is $30 \%$.(as shown in figure IV)

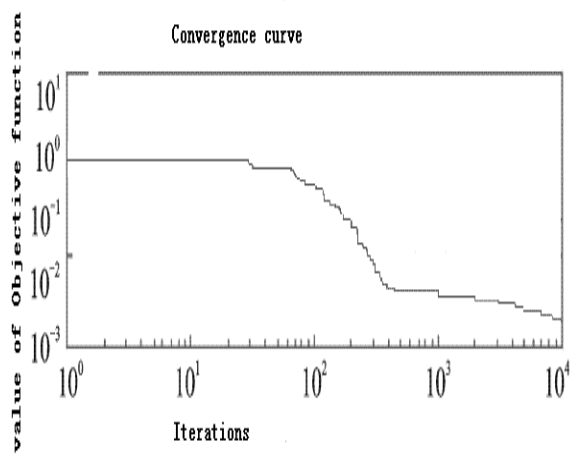

FIGURE III. CONVERGENCE CURVE OF CONDITION 1

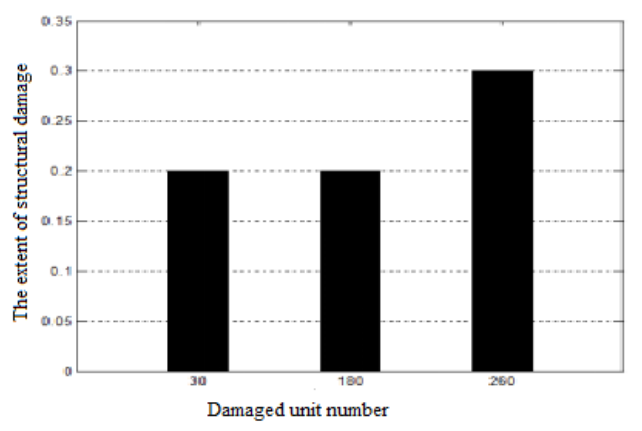

FIGURE IV. DAMAGE IDENTIFICATION RESULT

\section{Result Analysis}

Structural damage location and the extent of structural damage were effectively recognized by ACO algorithm and wavelet theory. This two-stage damage identification method combined the advantages of the two algorithms and avoided their own shortcomings. Blindness was reduced, the calculation efficiency was improved, the requirements of the computer hardware was reduced, and it has important implications for the calculation of complex structures.

\section{CONCLUSIONS}

1) A two-stage damage identification method combined by ACO algorithm and wavelet theory was put forward. This method can recognize structural damage location and extent effectively.

2) Structural finite element model was established. The curvature modal of structure were analyzed using continuous wavelet transform by Mexh wavelet on multiple scales. The location of the damage could be identified by the maximum of the wavelet coefficients. The objective function was established by the frequency and modal shape.ACO algorithm was used to solve constrained optimization problems, to identify the degree of structure damage.

3) This two-stage damage identification method put forward in this paper can greatly reduce the search space, reduce the hardware requirements of the computer, improve the computational efficiency, and it can be used for the identification of damage location and damage degree of complex engineering structures.

4) This method is proved to be verified by numerical analysis of a three-span continuous beam. It can be easily extended to damage identification of continuous beam bridge.

\section{ACKNOWLEDGMENT}

The work is supported by National Natural Science Foundation of China (No.51378079)

\section{REFERENCES}

[1] Liew K M,Wang Q,“Application of Wavelet Theory for Crack Identification in Structure" $[\mathrm{J}]$. Journal of Engineering, Mechanics, 1998,124(2):152-157.

[2] Raghavendrachar M, Akan A E . "Flexibility of Multi-reference Impact Testing for Bridge Diagnostics"[J].Journal of Structural Engineering, American Society of Civil Engineering, 1999,125(4):410- 416.

[3] M Rucka,K Wilde. "Application of Continuous Wavelet Transform in Vibration Based Damage Detection Method for Beams and Plates"[J].Journal of Sound and Vibration,2006,297(3-5):536-550

[4] Guan Deqing, Shi Li-cheng. "Damage Identification of Single Tower Cable-stayed Bridge Based on Wavelet Analysis of Curvature Mode"[J]. Journal of Architecture and Civil Engineering, 2010,27(1):21-42.

[5] Shi Li-cheng, Guan Deqing, Jiang Zhikai."Research on Damage of Continuous Steel Girders Identification by Wavelet Analysis of the Curvature Mode"[C].Proceedings of Sixth International Conference on Advances in SteelStructures.Hongkong:ICASS,2009:201-209.

[6] Dorigo M. "Optimization, Learning and Natural Algorithms" (in Italian) [D ]. Politecnicodi Milano, Italy, 1992.

[7] 'DorigoM, Gambardella L M. "Ant Colony System Cooperative Learning Approach to The Traveling Salesman Problem" [ J ]. IEEE Trans. Evolut.Comput., 1997, 1(1): 53-66. 
[8] Socha K. "ACO for continuous and mixed-variable optimization" [C]. Proceedings in ANTS 2004-Fourth International Workshop on Ant Colony Optimization and Swarm Intelligence . Brussels, Belgium, 2004.

[9] Gutjahr W J.A, "Graph-based Ant System and its Convergence.Future Generation Computer System,” 2000,6(8):873 888 